\title{
A NON-REGENERATIVE MODEL OF A REDUNDANT REPAIRABLE SYSTEM: BOUNDS FOR THE UNAVAILABILITY AND ASYMPTOTICAL INSENSITIVITY TO THE LIFETIME DISTRIBUTION
}

\author{
IGOR N. KOVALENKO \\ STORM Research Centre \\ University of North London \\ London $N 78 D B, U K$
}

(Received October, 1995; Revised February, 1996)

\begin{abstract}
In this paper we investigate steady state reliability parameters of an $F: r$-outof- $N$ redundant repairable system with $m(1 \leq m \leq r-1)$ repair channels in light traffic conditions. Such a system can also be treated as a closed queueing network of a simple kind. It includes two nodes, with infinite number of channels and $m$ channels, respectively. Each of the $N$ customers pass cyclically from one node to the other; the service time distributions are of a general form for both the nodes.

It is an $N$-component system with a general distribution $A(t)$ of free-of-failure periods of the components is considered. Failed components are repaired by an $m$-channel queueing system with a general distribution $B(t)$ of repair times. The system is assumed to be failed if and only if the number of failed components is at least $r$. (Only the rather difficult case $r \geq m+1$ is considered.)

Let $\mu$ be the intensity of the stationary point process of the occurrences of (partial) busy periods within which systems failures happen at least once, and let $Q$ be the steady-state unavailability of the system.

Two-sided bounds are established for $Q$ and $\mu$ based on the behavior of the renewal rate of an auxiliary renewal process. The bounds are used for deriving some asymptotical insensitivity properties in light traffic conditions.
\end{abstract}

Key words: Availability, Light Traffic, Perturbation Analysis, Redundancy, Repairable Systems, Non-regenerative Models, Fast Repair, Renewal Rate.

AMS (MOS) subject classifications: 60K10, 60K15, 60K25, 90B22, 90B25.

\section{Introduction}

Light traffic approximation is an important branch of queueing theory. For some of the most general results, see Baccelli and Schmidt [3] and Blaszczyszyn [4]; an excellent survey has been published by Blaszczyszyn et al. [5]. It should be mentioned that from the early sixties asymptotic methods of mathematical reliability have been extensively investigated in Russia. The theory having been developed can also be considered as contribution to light traffic theory. Soloviev [16] 
is the work of the most fruitful subsequent theory. For a recent presentation of the approach, see Soloviev [17] and Gertsbakh [6]. In an early paper by Kovalenko [9] a small parameter approach was suggested for the analysis of the availability of a complex system. For further results see Kovalenko [10-12], Pechinkin [14], Stadje [18], and Zazanis [19]. A small parameter approach was used for deriving variance reduction methods of the simulation of rare events; see Reiman and Weiss [15], Asmussen [1], Heidelberger et al. [8], Kovalenko and Kuznetsov [13], and Gnedenko and Kovalenko [7].

In this paper we investigate the behavior of a closed queue in light traffic conditions. The Markov process describing its behavior contains $m$ supplementary variables and thus cannot be reduced to a semi-Markov model. On the other hand, no parametric model (such as thinning or $\gamma$ dilation) is assumed. An interesting property is observed in the case of a closed queueing system: some parameters of the system are asymptotically (as traffic intensity $\rho \rightarrow 0$ ) insensitive to the distribution of free-of-failure time; only the mean free-of-failure time period is essential. A contrast can be observed in this point to open queues; see Atkinson [2].

\section{System Description}

Consider a system consisting of $N$ machines subject to random failures and repaired by $m$ repair channels. Usual i.i.d. and FIFO conditions are assumed to be satisfied. The following functions and parameters will be considered throughout the paper:

$A(t)$ - d.f. of a component's free-of-failure time: $\bar{A}(t)=1-A(t), \overline{\bar{A}}(t)=\int_{t}^{\infty} \bar{A}(x) d x ;$

$B(t)$ - d.f. of repair time: $\bar{B}(t)-1-B(t), \overline{\bar{B}}(t)=\int_{t}^{\infty} \bar{B}(x) d x$

$\varphi(z)=\int e^{z t} d B(t)$

$h(t)$ - renewal rate of a renewal process formed by points $\xi_{0}, \xi_{0}+\eta_{0}+\xi_{1}, \xi_{0}+\eta_{0}+\xi_{1}+\eta_{1}+$ $\xi_{2}, \ldots$, where $\xi_{i}$ and $\eta_{i}$ are totally independent and distributed according to $\xi_{i} \sim A(t)$, $\eta_{i} \sim B(t)$

$h_{U}(t)=\sup _{x \geq t} h(x), h_{L}(t)=\inf _{x \geq t} h(x), \lambda=h_{U}(0), \Lambda=N \lambda$.

The time scale is assumed to be chosen in such a way that

$$
\int_{0}^{\infty} t d B(t)=\rho, \quad \int_{0}^{\infty} t d A(t)=1-\rho ; 0<\rho<1 .
$$

The following Markov process $\zeta(t)$ will be considered for describing the system's behavior:

$$
\zeta(t)=\left(\xi_{i}(t), \eta_{i}(t), 1 \leq i \leq N\right)
$$

where $\xi_{i}(t)$ is the residual time to failure of machine $i$ if it is operating at time $t$; $\xi_{i}(t)$ is not defined otherwise; $\eta_{i}(t)$ is the residual repair time of machine $i$ if it is being repaired; $\eta_{i}(t)$ is not defined otherwise. [Thus, neither $\xi_{i}(t)$ nor $\eta_{i}(t)$ is defined for a queueing machine.]

In what follows it is assumed that the process $\zeta(t)$ and some point processes connected to it that are required for our purposes are stationary. Our aim consists of the derivation of upper and lower bounds for the following parameters:

$\mu$ - intensity of (partial) busy periods within which the queue length $\nu(t)$ achieves a fixed level $r$ at least once $(m+1 \leq r \leq N)$; 
$Q$ - steady state probability of the event $\{\nu(t) \geq r\}$, i.e., the unavailability of the system.

Both the upper and the lower bounds are obtained by a straightforward renewal argument. The following properties are used: a compactness property of the process $\zeta(t)$ yielding some bounds for $\zeta(0)$, analysis of busy periods in a time interval $(0, T)$, analysis of a virtual busy period starting at time $T$, and analysis of the renewal probability of all machines in the interval $(0, T)$. Essentially, the local independence of alternating renewal behaviors of machines as soon as $\nu(t)<m$ is used.

\section{Upper Bounds}

\subsection{Ergodic bounds}

The following inequalities hold true:

$$
P\left\{\xi_{i}(0) \in d x\right\} \leq \bar{A}(x) d x, \quad P\left\{\eta_{i}(0) \in d x\right\} \leq \bar{B}(x) d x .
$$

In particular,

$$
P\left\{\xi_{i}(0) \geq x\right\} \leq \overline{\bar{A}}(x), \quad P\left\{\eta_{i}(0) \geq x\right\} \leq \overline{\bar{B}}(x)
$$

and

$$
P\{\nu(0) \geq 1\} \leq N \rho \text {. }
$$

\subsection{Upper bounds for the factorial moment intensity}

For a fixed $T>0$, machine $i$ called $T$-renewed if $\xi_{i}(0)<T$ or if $\xi_{i}(0)$ is not defined. Denote by $h_{T}^{(s)}\left(x_{1}, \ldots, x_{s-1}\right) \delta^{s}+o\left(\delta^{s}\right)$ the probability of such an event: $\quad$ all the machines are $T$ renewed; some different machines fail during time intervals $(T, T+\delta),\left(T+x_{1}, T+x_{1}+\delta\right),(T+$ $\left.\left.x_{2}, T+x_{2}+\delta\right), \ldots,\left(T+x_{s-1}, T+x_{s-1}+\delta\right), 0<x_{1}<\ldots<x_{s-1}, \delta \rightarrow 0\right\}$.

Consider the expressions

$$
H_{1}^{(s)}=N^{[s]} \lambda^{s} \varphi_{0}\left(z_{0}\right)\left(\left(1-\left(\Lambda \varphi_{0}\left(z_{0}\right) \varphi\left(2 N z_{0}\right) / z_{0}\right)\right)^{-1} \exp \left\{\Lambda^{m+1} T \varphi^{m}(z) /\left(z^{m}-\Lambda z^{m-1} \varphi(z)\right)\right\}-1\right)
$$

where

$$
\varphi_{0}(z)=\left(1+\frac{1}{2} N^{2} z \varphi^{\prime \prime}(N z)\right) \varphi^{N}(z)
$$

provided $z$ and $z_{0}$ are positive and the denominators are positive as well, and

$$
H_{0}^{(s)}=N^{[s]}\left(h_{U}^{s}\left(T-T_{0}\right)+\lambda^{s}\left(N \overline{\bar{A}}\left(T_{0}-\Delta\right)+N^{2} \rho \bar{A}\left(T_{0}-\Delta\right)+e^{-z_{1} \Delta} \varphi_{0}\left(z_{0}\right)\right)\right) .
$$

Then the following upper bound holds true:

$$
h_{T}^{(s)}\left(x_{1}, \ldots, x_{s-1}\right) \leq H_{0}^{(s)}+H_{1}^{(s)}
$$

for every positive $z, z_{i}$; the denominators are positive as well.

We also have 


$$
h_{T}^{(s)}(\ldots) \leq\left(H_{1}^{(0)}+1\right) N^{[s]} \lambda^{s} \leq\left(H_{1}^{(0)}+1\right) \Lambda^{s}
$$

\subsection{Upper bound for $\mu$}

We have

$$
\mu \leq \mu_{0}+\mu_{1}+\mu_{2}
$$

where

$$
\mu_{0}=\left\{\begin{array}{cc}
\frac{1}{(r-1) !}\left(H_{0}^{(r)}+H_{1}^{(r)}\right) \rho^{r-1} & \text { if } r=m+1 \\
\frac{1}{m !(r-m-2) !}\left(H_{0}^{(r)}+H_{1}^{(r)}\right) \int_{0}^{\infty} x^{r-m-2} \overline{\bar{B}}^{m}(x) d x & \text { if } r \geq m+2 \\
\mu_{1}\left(H_{1}^{(0)}+1\right) \Lambda^{r+1}(2 \varphi(z) / z)^{r} & \\
\mu_{2}=N \bar{A}(T) . &
\end{array}\right.
$$

\subsection{Upper bound $Q$}

We have

$$
Q \leq Q_{0}+Q_{1}+Q_{2}+Q_{3}
$$

where

$$
\begin{gathered}
Q_{0}=\frac{1}{m !(r-m-1) !}\left(H_{0}^{(r)}+H_{1}^{(r)}\right) \int_{0}^{\infty} x^{r-m-1} \overline{\bar{B}}^{m}(x) d x \\
Q_{1}=(r+1)\left(H_{1}^{(0)}+1\right)(\Lambda / z)^{r+1}(2 \varphi(z))^{r} / \psi^{2} \\
Q_{2}=N \bar{A}(T)\left(H_{1}^{(0)}+1\right) \varphi^{N}(z) /\left(z \psi^{2}\right) \\
Q_{3}=2 N \bar{A}(T)\left(H_{1}^{(0)}+1\right) \Lambda \varphi^{N+1}(z) /\left(z^{2} \psi^{3}\right)
\end{gathered}
$$

with

$$
\begin{aligned}
& \psi=1-2 \Lambda \varphi(z) / z \\
& z>0,2 \Lambda \varphi(z)<z
\end{aligned}
$$

\section{Lower Bounds}

\subsection{Lower bound for the joint p.d.f.}

For $0<x_{1}<\ldots<x_{r+1}$, denote by $f\left(x_{1}, \ldots, x_{r-1}\right) \delta^{r}+o\left(\delta^{r}\right)$ the (T-independent) probability of the event $\left\{\nu(T)=0 ; \quad 0<\xi_{i_{1}}(T)<\delta, \ldots, x_{r-1}<\xi_{i_{r}}<x_{r-1}+\delta\right.$ for some $i_{1}, \ldots, i_{r}$; $\xi_{i}(T)>x_{r-1}$ for all other $\left.i\right\}$. Then we have, for $0<\Delta<T_{0}{ }^{r}<T$,

$$
f\left(x_{1}, \ldots, x_{r-1}\right) \geq N^{[r]}(1-\epsilon)\left(c_{0}-c_{1} x_{r-1}\right),
$$


where

$$
\begin{gathered}
\epsilon=N\left(\rho(1+1 / \Delta)+\overline{\bar{A}}\left(T_{0}-\Delta\right)\right), \\
c_{0}=h_{L}^{r}\left(T-T_{0}\right)-\lambda^{r+1}\left(\left(N^{2}-r\right) \rho+N \Delta+\left(\begin{array}{c}
N \\
m
\end{array}\right)(N-m) T(\lambda \rho)^{m}\right), \\
c_{1}=N \lambda^{r+1} .
\end{gathered}
$$

\subsection{Lower bounds for $\mu$}

We have

$$
\mu \geq N^{[r]}(1-\epsilon)\left(c_{0} i_{0}-c_{1} I_{1}\right),
$$

where

$$
\begin{aligned}
& I_{0}=\left\{\begin{array}{cl}
\frac{1}{(r-1) !} \rho^{r-1} & \text { if } r=m+1 \\
\frac{1}{m !(r-m-2) !} \int_{0}^{\infty} x^{r-m-2} \overline{\bar{B}}^{m}(x) d x & \text { if } r \geq m+2
\end{array}\right. \\
& I_{1}=\frac{\rho^{m-2}}{(m-2) !(r-m+2) !(r-m)} \int_{0}^{\infty} x^{r-m+2} d B(x)
\end{aligned}
$$

\subsection{Lower bound for $Q$}

We have

$$
Q \geq N^{[r]}(1-\epsilon)\left(c_{0} J_{0}-c_{1} J_{1}\right)
$$

where

$$
\begin{gathered}
J_{0}=\frac{1}{m !(r-m-1) !} \int_{0}^{\infty} x^{r-m-1} \overline{\bar{B}}^{m}(x) d x, \\
J_{1}=\frac{1}{(m-2) !(r-m) !(r-m+2)(r-m+3)} \rho^{m-2} \int_{0}^{\infty} x^{r-m+3} d B(x) .
\end{gathered}
$$

\section{Proof of the Bounds}

In this section, routine transformations are omitted; the discussion of the principal moments should still be comprehensible.

1. For a given $i$, the measure of the set of points of an interval $\left(0, t^{\prime}\right)$ for which, say, $\xi_{i}(t) \in$ $d x$ coincides with a similar measure for an alternative renewal process, but for a shorter time interval $\left(0, t^{\prime \prime}\right)$. To derive (1)-(3) one must only average.

2. Define a busy period in the obvious way. Call it blocked if at least $m+1$ repairs are commenced within it. A busy period will be called a failure if $\nu(t) \geq r$ at least once within it. The first repair of each machine overlapping the interval $(0, T)$ will be said to be initial. Denote by $Y$ 
the total length of initial repairs within the interval $(0, T)$. A busy period will be called initial if it includes an initial repair. Thus the number of initial busy periods can vary from 0 to $N$.

The $s$-dimensional factorial intensity $h_{T}^{(s)}$ can be conceived as the sum of intensities, say, $g_{n}\left[i_{0}, \ldots, i_{n}\right]$ where $n$ is any possible number of non-initial busy periods, $i_{k}+m+1$ is the number of repairs within the $k$ th of them if $k \geq 1$, and $i_{0}$ is the number of non-initial repairs within initial busy periods in total.

Let $S_{k}$ be the sum of $k$ independent r.v. with the d.f. $B(t)$. For a given $i_{k}, k \geq 1$, the duration of the $k$ th blocked busy period does not exceed $S_{i_{k}+m+1}$. Having chosen $i_{k}$ and associated $S_{i_{k}+m+1}$ we must choose $n(m+1)=i_{0}+\ldots+i_{n}$ random points of non-initial failures. For each machine we thus obtain a chain from its initial failure to its last one within the interval $(0, T)$ or to a point in the set $\left\{T, T+x_{1}, \ldots, T+x_{r-1}\right\}$. There can be gaps in chains due to the queueing. Only the following properties are used.

(i) the density of each jump from $t$ to $t^{\prime}$ for a given machine does not exceed $\lambda$, and

(ii) for $i_{l}=\ell$ at least $\ell-1$ customers must arrive within the interval $S_{\ell-1}$ (though the $S_{\ell-1}$ should be changed to $Y+N S_{\ell}$ for the totality of initial busy periods).

The bound (4) can be obtained by the summation over $n ; i_{0}, \ldots, i_{n}$ (unless $n=i_{0}=0$ ), integration over an admissible region, and the application of moment generating functions (mg.f.). The bound (5) for the m.g.f. on $Y$ is based on the stochastic inequality

$$
Y \leq S_{n}+\eta_{1}(0)+\ldots+\eta_{N}(0)
$$

making use of (1) to (3).

The case $n=i_{0}=0$ is discussed separately. The bound (6) for $g_{0}[0]$ is implied from the statement that if $\xi_{i}(0)<T_{0}-\Delta$ and $\sum_{i} \eta_{i}(0)<\Delta$ then all the first renewals occur up to time $T_{0}$ and thus $h_{U}\left(T-T_{0}\right)$ will bound the renewal rate for each of the chosen $s$ machines at points $T, T+x_{j}$. A rougher bound (8) is obtained as soon as the case $n=i_{0}=0$ is not separated.

3. The bound (9) becomes clear if it is noted that $\mu_{0}$ is a bound for the intensity of failure busy periods with monotone system failures; $\mu_{1}$ bounds the intensity of busy periods with at least $r+1$ repairs, whereas $\mu_{2}$ accounts for non- $T$-renewed failures, no such failure being included in $\mu_{0}$ or $\mu_{1}$. The bounds (9) to (11) are familiar from reliability theory of repairable systems though we use renewal intensities instead of p.d.f. The bound (12) follows from ergodic reasoning: the intensity of the point process of busy periods of the kind being considered does not exceed the intensity of happenings $\{$ time to failure exceeds $T$ \}.

The bounds (13) to (17) are derived in a similar way. The bound (14) counts mean sojourn time in failure states after a monotonic failure; (15) bounds the mean length of a busy period with at least $r+1$ failures; (16) counts the lengths of busy periods originated by the failure of a non- $T$-repaired machine; finally, (17) is due to the possibility of such failures afterwards. Some combinatorics is used, in particular, the bound $\left(H_{1}^{(0)}+1\right)(2 \Lambda t)^{k} / k$ ! for the probability of at least $k$ failures (including repeated ones) in an interval $(T, T+t)$.

Note that originally we have obtained upper bounds without referring to m.g.f. but basing them on some infinite series for the moments of r.v. $S_{k}$; we omit the details.

The lower bound (20) is obtained from another idea. Let the event $B$ consist of nno failure machine at time 0$\}$ and $\left\{\right.$ all times to failures are less than $\left.T_{0}-\Delta\right\}$ and $\{$ total time of first repairs is less than $\Delta\}$. If the event $B$ occurs then all the renewals occur at points $t_{i}^{\prime}<T_{0}$ if no queueing happens afterwards.

Let us make independent alternating renewal processes to start from the points $t_{i}^{\prime}$. Denote by 
$\nu^{*}(t)$ the number of down-phases of these processes at time $t$. If, moreover,

(i) some "independent" failures of different machines occur in time intervals $(T, T+\delta), \ldots$, $\left(T, x_{r-1}, T+x_{r-1}+\delta\right)$;

(ii) no repeated failure occurs in the interval $\left(T, T+x_{r-1}+\delta\right)$;

(iii) the remaining $N-s$ machines are operating in the interval $\left(T, T+x_{r-1}+\delta\right)$,

(iv) $\nu^{*}(t) \leq m$ for $t \in(0, T)$ with possible exception of initial repairs; and

$(v)$ no non-initial repair overlaps an initial busy period,

then the considered situation for the intervals $(T, T+\delta), \ldots,\left(T+x_{r-1}, T+x_{r-1}+\delta\right)$ and intermediate intervals holds for the original (dependent) queueing process as well. The analysis of the main event and of possible exceptions cautioned above, $(i i)$ to $(v)$, directly leads to the lower bound (20). As to (21), $\epsilon$ bounds the probability of the event complementary to $B$.

The bounds (24) to (29) are obtained via the integration of the inequality (20) with the weight $\Pi \bar{B}(\ldots)$ over the region associated with monotone failures.

\section{Asymptotical Insensitivity}

Denote $\mu=\mu[A, B], Q=Q[A, B]$ by $W=W[A, B]$ any system parameter, and introduce the parameters

$$
V_{U}\left[\mathcal{A}_{\rho}, \mathscr{B}_{\rho}\right]=\sup _{B \in \mathscr{B}_{\rho}} \sup _{A \in \mathcal{A}_{\rho}} W[A, B] / W\left[E_{1-\rho}, B\right],
$$

for any classes of distributions containing $E_{1-\rho}:=1-e^{-t /(1-\rho)}$ and $E_{\rho}:=1-e^{-t / \rho}$ respectively. If for a system parameter $W$ (particularly for $\mu$ or $Q$ ) the relation $V_{U}\left[\mathcal{A}_{\rho}, \mathfrak{B}_{\rho}\right] \underset{\rho \rightarrow 0}{\rightarrow} 1$ holds, then we will say that the parameter $W$ is insensitive to the input distribution from $\stackrel{\rho}{\rho} \overrightarrow{b o v e}$ w.r.t. the family $\left(\mathcal{A}_{\rho}, \mathscr{B}_{\rho} ; \rho>0\right)$. The asymptotical insensitivity from below will be defined by the relation

$$
V_{L}\left[\mathcal{A}_{\rho}, \mathscr{B}_{\rho}\right]=\inf _{B \in \mathscr{B}_{\rho}} \inf _{A \in \mathcal{A}_{\rho}} W[A, B] / W\left[E_{1-\rho}, B\right] \underset{\rho \rightarrow 0}{\rightarrow} 1
$$

In a similar way, the asymptotical insensitivity from above (below) to the repair distribution can be defined, but it will not be discussed in this paper.

Asymptotical insensitivity can be defined as the conjunction of those from above and from below. Note that in practical analysis, very often a one-sided bound is of interest.

Introduce the following conditions:

(A1) There exists a function $z_{\rho}>0$ such that the ration $\alpha_{\rho}:=\varphi\left(z_{\rho}\right) / z_{\rho} \rightarrow 0$ as $\rho \rightarrow 0$ and $\varphi(z \rho) \leq \theta$, a constant.

(A2) There exists a function $T=T_{\rho}$ such that $T \alpha_{\rho}^{m} \rightarrow 0$ and $\overline{\bar{A}}(T) \rightarrow 0$ as $\rho \rightarrow 0$.

(A3) For the same function $T=T_{\rho}, h_{U}(T) \rightarrow 1$ as $\rho \rightarrow 0$.

(A4) For the same function $T=T_{\rho}, \bar{A}(T)=o\left(\rho^{r-1}\right)$.

(A5) For the same function $T=T_{\rho}, \bar{A}(T)=o\left(\rho^{r} z_{\rho}\right)$.

All the limits in (A1) to (A5) should be considered in the uniform sense w.r.t. a family $\left(A_{\rho}, B_{\rho}, \rho>0\right)$. 
$A(t)=A_{\rho}(t), B(t)=B_{\rho}(t)$ are any d.f. from the classes $\mathcal{A}_{\rho}$, resp. $\mathscr{B}_{\rho} ; \overline{\bar{A}}(0)=1-\rho, \overline{\bar{B}}(0)=$ $\rho, \varphi(z)=\varphi_{\rho}(z)=\int_{0}^{\infty} e^{z t} d B_{\rho}(t)$.

Theorem 1: The conditions $(A 1)-(A 4)$ in totality are sufficient for the asymptotical insensitivity of $\mu$ from above. The conditions $(A 1)-(A 3)$ and $(A 5)$ in totality are sufficient for the asymptotical insensitivity of $Q$ from above.

Proof: Reduces to the investigation of the behavior of upper bounds stated in section 3 . Only the point $z=z_{\rho}$ is considered while investigation $\varphi(z)$. For $\varphi_{0}(z)$ the bound $(1+$ $\varphi(2 N z) / z) \varphi^{N}(z), z>0$ can be obtained by contour integration, thus while investigating $H_{1}^{(r)}$, one can set $z_{0}=z_{\rho} /(2 N p), p>1$. Making use of the convexity of $\varphi(z), z>0$, one obtains $\varphi\left(z_{0}\right) \leq$ $1+(\theta-1) /(2 N p)$; we also have $\varphi\left(2 N z_{0}\right) / z_{0} \leq 2 N \alpha_{\rho} p$. Since $p$ is arbitrary, the relation $H_{1}^{(r) \rightarrow 0}$ holds as $\rho \rightarrow 0$.

For establishing the asymptotical insensitivity from below, some new assumptions should be introduced in which all the limits are uniform w.r.t. the family $\left(\mathcal{A}_{\rho}, \mathscr{B}_{\rho}, \rho>0\right)$.

(A6) There exists a function $T^{\prime}=T_{\rho}^{\prime}$ such that $T^{\prime} \rho^{m} \rightarrow 0, \overline{\bar{A}}\left(T^{\prime}\right) \rightarrow 0$, and $h_{L}\left(T^{\prime}\right) \rightarrow 1$ as $\rho \rightarrow 0$.

(A7) $\int_{0}^{\infty} t^{r-m+2} d B(t)=o\left(\rho^{r-m+1}\right), \rho \rightarrow 0$.

$$
\int_{0}^{\infty} t^{r-m+3} d B(t)=o\left(\rho^{r-m+2}\right), \rho \rightarrow 0 .
$$

Theorem 2: The totality of conditions $(A 6),(A 7)$ imply the asymptotical insensitivity of $\mu$ from below. The totality of conditions $(A 6),(A 8)$ imply the asymptotical insensitivity of $Q$ from below.

Proof: A straightforward application of the bounds from section 4 . One should set $T_{0}:=$ $T^{\prime}+\sqrt{\rho}, \Delta=\sqrt{\rho}, T-T_{0}:=T^{\prime}$.

\section{Acknowledgement}

The result has been reported as parts of the author's talks in the Workshop on Stochastic Networks (August 1995, Edinburgh) and the XXIV All-Polish Conference on Applications of Mathematics (September 1995, Zakopane-Kośćielisko). Many thanks to Professors F. Baccelli, S.G. Foss, V.S. Korolyuk, T. Rolski and A.D. Soloviev, whom I met there, for their valuable discussions with me. The author is very much grateful to Professor J.H. Dshalalow for the support of the paper and to Mr. Gary Russell for his careful editing and improving it linguistically.

\section{References}

[1] Asmussen, S., Light traffic equivalence in single-server queues, Ann. Appl. Prob. 2 (1992), 555-574.

[2] Atkinson, J.B., The transient M/G/1/0 queue: Some bounds and approximations for light traffic with application to reliability (a manuscript), School of Mathematical Sciences, University of North London 1995.

[3] Baccelli, F. and Schmidt, V., Taylor expansions for Poisson driven (max, + )-linear systems, Research Report 2494, INRIA (1995).

[4] Blaszczyszyn, B., Factorial moment expansion for functionals of point processes with applications to approximations of stochastic models (a doctoral dissertation), Math. Institute, Wroclaw University, Wroclaw, Jan. 1995. 
[5] Blaszczyszyn, B., Rolski, T. and Schmidt, V.., Light traffic approximations in queues and related stochastic models, Advances in Queueing, (ed. by J. Dshalalow), CRC Press, Boca Raton, Florida 1995, 379-406.

[6] Gertsbakh, I.B., Asymptotic methods in reliability theory: A review, Adv. Appl. Prob. 16 (1984), 147-175.

[7] Gnedenko, B.V. and Kovalenko, I.N., Introduction to Queueing Theory, Birkhäuser, Boston 1989.

[8] Heidelberger, P., Shahabuddin, P. and Nicola, F., Bounded relative error in estimating transient measures of highly dependable nonMarkovian systems, Chapter 18 in Reliability and Maintenance of Complex Systems, Lecture Notes, NATO ASI, June 12-22, 1995, Kemer, Antalya - Turkey 1995.

[9] Kovalenko, I.N., On some problems of complex systems reliability, Kibernetika na Sluzhbu Kommunizma 2 (ed. by A.I. Berg and B.V. Gnedenko), Energia, Moscow (1964), 194-205 (in Russian).

[10] Kovalenko, I.N., Rare Event Analysis in Estimation of Systems Efficiency and Reliability, Sov. Radio, Moscow 1980 (in Russian).

[11] Kovalenko, I.N., Rare events in queueing systems - A survey, Queueing Sys. 16:1 (1994), 149.

[12] Kovalenko, I.N., Approximations of queues via small parameter method, Advances in Queueing, (ed. by J. Dshalalow), CRC Press, Boca Raton, Florida 1995, 481-506.

[13] Kovalenko, I.N. and Kuznetsov, N.Yu., Methods of High Reliable Systems Analysis, Radio i Svyaz, Moscow 1988 (in Russian).

[14] Pechinikin, A.V., The analysis of one-server systems with small load, Eng. Cybern. 22 (1984), 129-135.

[15] Reiman, M.I. and Weiss, A., Light traffic derivatives via likelihood ratios, IEEE Trans. on Inf. Theory 35:3 (1989), 648-654.

[16] Soloviev, A.D., Asymptotic behavior of the time of the first occurrence of rare events in a regenerative process, Eng. Cybern. 9:6 (1970), 79-89.

[17] Soloviev, A.D., Asymptotic methods for high reliable repairable systems, Chapter 6 in Handbook of Reliability Engineering, (ed. by I.A. Ushakov and R. Harrison), USA 1993, 113-137.

[18] Stadje, W., A new approach to the distribution of the duration of the busy period for G/G/1 queueing system, J. Austral. Math. Soc. Ser. A 48 (1990), 89-100.

[19] Zazanis, M.A., Analyticity of Poisson-driven stochastic systems, Adv. Appl. Prob. 24 (1992), 532-541. 


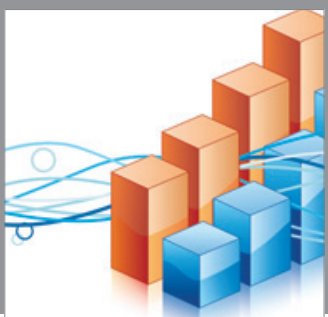

Advances in

Operations Research

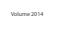

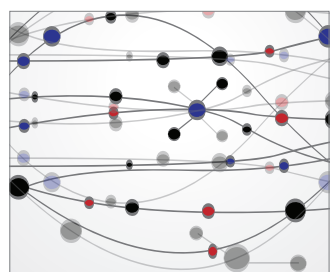

\section{The Scientific} World Journal
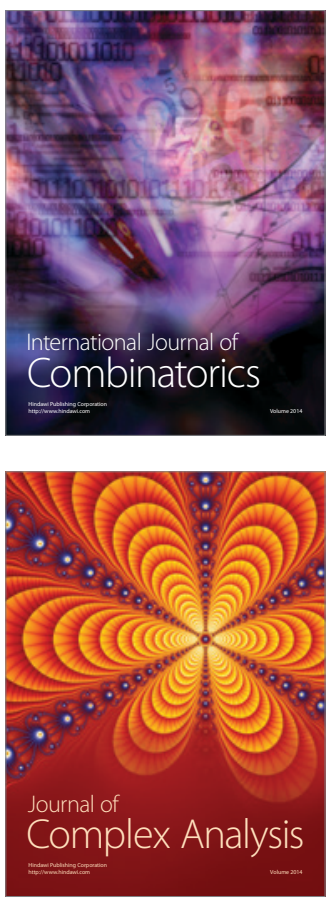

International Journal of

Mathematics and

Mathematical

Sciences
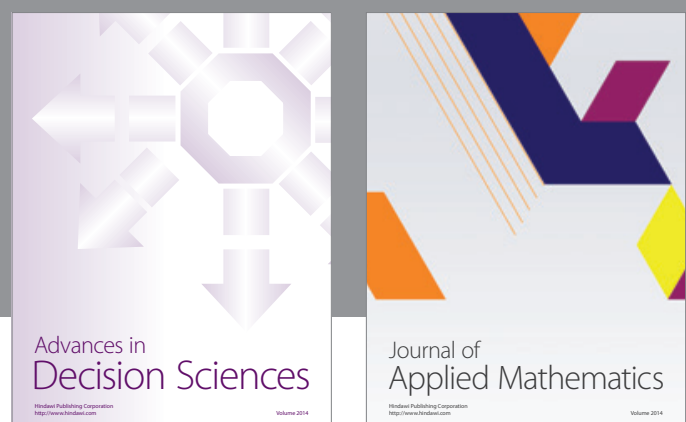

Journal of

Applied Mathematics
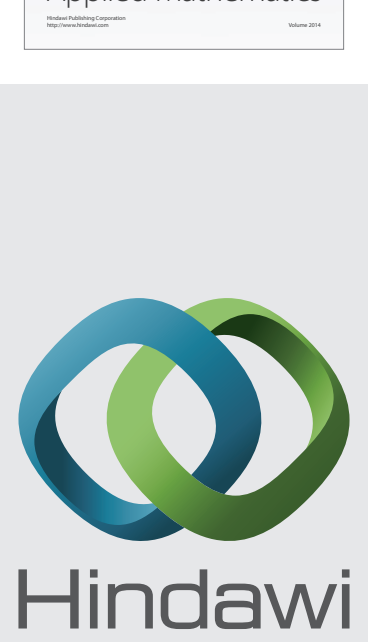

Submit your manuscripts at http://www.hindawi.com
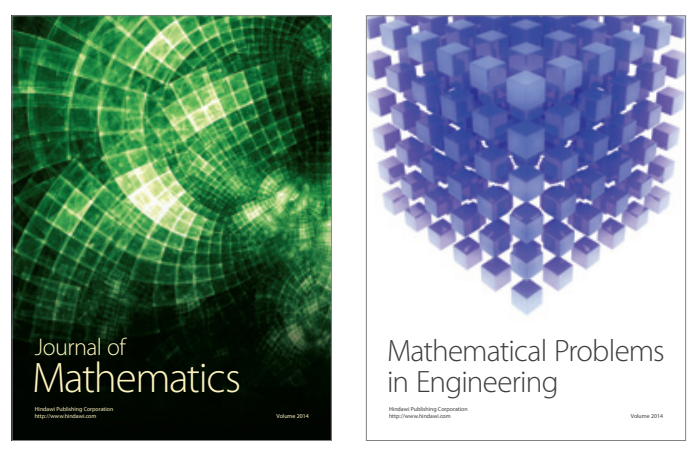

Mathematical Problems in Engineering
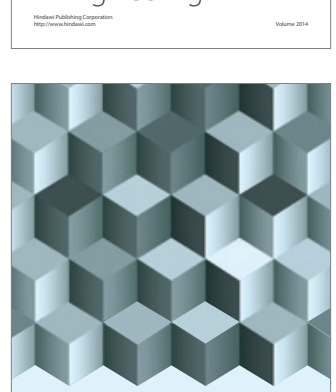

Journal of

Function Spaces
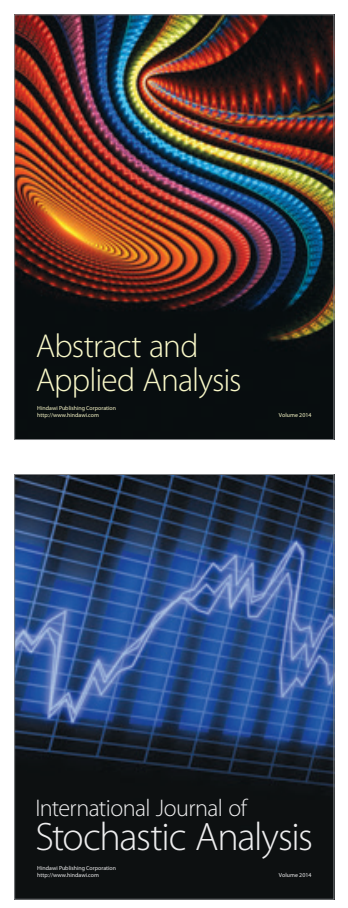

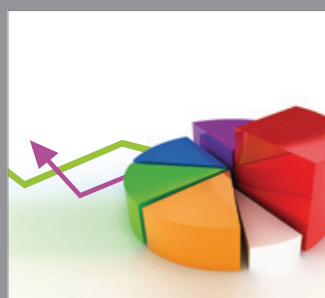

ournal of

Probability and Statistics

Promensencen
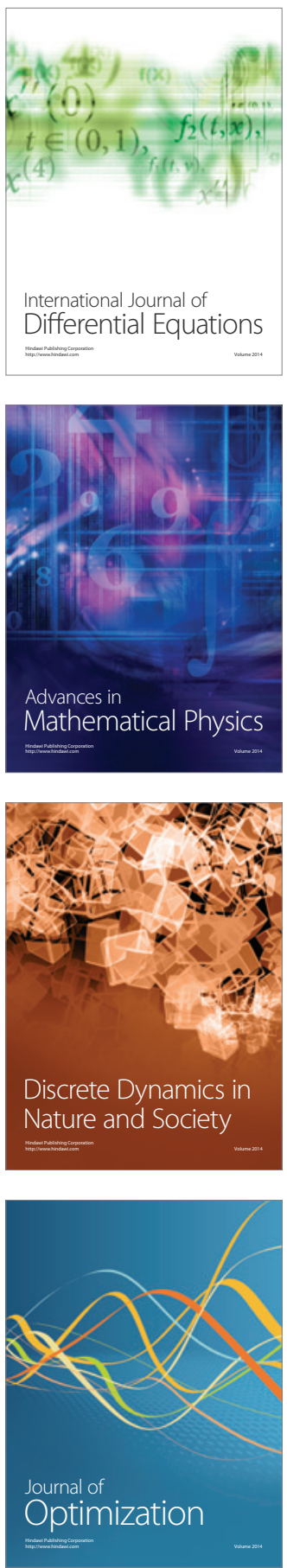